\title{
Exercício físico e avaliação da capacidade funcional de um idoso com Parkinson
}

\author{
Physical exercise and evaluation of the functional capacity of an elderly with \\ Parkinson
}

Lucas Messias Pinto Araújo ${ }^{1 *}$, Ana Patrícia Guimarães Rodrigues ${ }^{1}$

ARTIGO ORIGINAL | ORIGINALARTICLE

O objetivo desse estudo foi verificar a associação de um Programa de Exercício Físico (PEF) com a capacidade funcional de um idoso acometido pela Doença de Parkinson (DP). Esta pesquisa teve um participante do sexo masculino, 70 anos, caracterizando um estudo de caso. O PEF foi realizado duas vezes por semana, 45 minutos de duração, durante 27 meses. Os testes funcionais realizados foram: velocidade de reação, equilíbrio estático e sit-and-reach. Foram realizadas seis avaliações funcionais periodicamente após o início das atividades. Para a análise dos dados foi utilizado o teste de correlação Spearman. Nos testes de velocidade de reação os resultados apresentaram força de correlação positiva fraca $(+0.14)(p=0.78)$. Nos testes de equilíbrio os resultados apresentaram força de correlação positiva fraca $(+0.25)(p=0.62)$. Nos testes sit-and-reach, os resultados com a perna direita apresentaram força de correlação positiva fraca (+ $0.23)(p=0.65)$ e com a perna esquerda, força de correlação inexistente $(0.00)(p=1.00)$. De acordo com os resultados apresentados, não houve associação significativa do PEF com a capacidade funcional do idoso. Palavras-chave: educação física, doença crônica, doença de Parkinson, aptidão física, atividade motora.

\begin{abstract} The goal of this study was to verify the association of the Physical Exercise Program (PEF) with the Functional of an Elderly person performed by Parkinson's Disease (PD). This research had a 70-year-old male sexual component, characterizing a case study. The PEF was performed twice a week, 45 minutes long, for 27 months. The healthy tests were: reaction velocity, static equilibrium and sit-and-reach. Six functional evaluations were performed periodically after the start of activities. Spearman's correlation test was used to analyze the data. In the tests of reaction speed the obtained results presented weak positive agitation $(+0.14)(p=0.78)$. In the static equilibrium test, the results were positive $(+0.25)(p=0.62)$. In the sit-and-reach test, the results with the right leg offer of a positive offer $(+0.23)(p=0.65)$, and the one left leg presented nonexistent coordination force $(0.00)(p=1.00)$. According to the results presented, there was no significant association of PEF with the functional capacity of the elderly.

Keywords: physical education, parkinson's disease, chronic disease, physical aptitude, motor activity.
\end{abstract}

\footnotetext{
${ }^{1}$ Universidade Estadual Vale do Acaraú, Sobral, Brasil

* Autor correspondente: lucasmessias.2012@hotmail.com
} 


\section{INTRODUÇÃo}

A doença de Parkinson (DP) foi primeiramente descrita em 1817 por James Parkinson, que a nomeou paralisia agitante, baseado em seus sintomas: bradicinesia, rigidez muscular e principalmente tremor de repouso com marcha típica (Limongi, 2001).

A DP é decorrente da incapacidade de uma parte dos neurônios que estão localizados na substancia negra produzirem dopamina. Tal patologia compromete gravemente as funções motoras, com uma evolução que pode levar à forma rígido acinética em questão de poucos anos (Guyton \& Hall, 2017).

A DP acomete cerca de $0.1 \%$ da população mundial e $1 \%$ da população acima de 65 anos. A chance de os indivíduos serem acometidos cresce na medida que envelhecem (Galhardo, Amaral \& Vieira, 2009). Devido ao aumento da expectativa de vida, estudos apontam que mais de 40.000 .000 de indivíduos no ano de 2020, terão DP (Morris, 2000). De acordo com Souto (2009), o Brasil será em 2030, o quinto país a ter o maior número de pessoas acometidas pela doença, aproximadamente 340 mil pessoas.

As pesquisas na área de Biologia Molecular e Patologia avançaram nas últimas décadas (Petzinger et all., 2015; Burke, Dauer \& Vonsattel, 2008), contudo existe uma urgência em compreender melhor a influência da atividade física regular na evolução da DP, já que o tratamento físico tem se mostrado promissor (Borrione, Tranchita, Sansone, \& Parisi, 2014; Rose, Løkkegaard, Sonne-Holm, \& Jensen, 2013).

Ainda não há consenso de um protocolo de atividade física para o tratamento da doença. De acordo com Gobbi, Barbieri e Vitório (2014), são poucas as pesquisas que utilizam um programa de atividade física de longa duração com os indivíduos com DP. O objetivo desse estudo foi verificar a associação de um Programa de Exercício Físico (PEF), realizado em um período de 27 meses, com a capacidade funcional de um idoso com DP.

\section{MÉTODO}

A pesquisa é caracterizada como estudo de caso. De acordo com Chehuen (2012) o estudo de caso é um tipo de pesquisa na qual o investigador estuda de forma minuciosa o objeto de investigação.

\section{Participantes}

Um indivíduo do sexo masculino com 70 anos de idade participou da pesquisa, inicialmente com nível dois da doença, segundo a Escala de Hoehne Yahr (HY - Degree of Disability Scale) (Hoehn \& Yahr, 1967). As características iniciais foram: sintomas bilaterais, disfunção mínima e comprometimento da postura e marcha. Ao final do estudo, passados dois anos, evoluiu para nível três da doença, cuja as características foram: sintomas bilaterais, aumento da instabilidade postural, dificuldade de equilíbrio e marcha.

$O$ indivíduo nasceu em Sobral-CE, tem o hábito de exercitar-se esporadicamente. Diariamente realiza cinco refeições. Nunca fumou, é obeso, hipertenso, diabético e toma os medicamentos necessários para a sua condição.

A pesquisa foi realizada atendendo as normas 466/12 do Conselho Nacional de Saúde (CNS) (2013), a qual trata dos procedimentos éticos em estudos envolvendo seres humanos. O projeto foi submetido e aprovado no comitê de ética e pesquisa da Universidade Estadual Vale do Acaraú, com CAAE 51679115.3.0000.5053. O indivíduo concordou em participar da pesquisa e assinou o termo de consentimento livre e esclarecido.

\section{Instrumentos e Procedimentos}

A anamnese (Rocha \& Guedes, 2013) e todos os testes funcionais foram realizados no laboratório de Fisiologia do Esforço (LAFESF) da Universidade Estadual Vale do Acaraú - UVA, na cidade de Sobral-CE. Foram realizados exames médicos e laboratoriais para garantir a segurança do indivíduo.

Após responder ao questionário de anamnese (Rocha \& Guedes, 2013) o indivíduo participou do PEF, às quartas e sextas feiras, durante 45 minutos, ao longo de 27 meses. Quatro meses após o início foi realizada a primeira avaliação funcional. As demais avaliações foram realizadas, respectivamente, 9, 13, 16, 19 e 27 meses após o início. 
Os testes realizados foram, Velocidade de Reação (VR) (Rocha \& Guedes, 2013), Equilíbrio Estático (EE) (Spirduso, Francis \& Macrae, 2005) e o sit-and-reach (SR) (Jones, Rikli, Max \& Noffal, 1998). Estes, foram realizados durante a fase "on", referente ao uso dos medicamentos, facilitando a realização dos movimentos pelo indivíduo.

Para a realização do teste de VR o avaliado ficou sentado em uma cadeira, com as mãos na cintura, os pés juntos e olhando para frente. Ao sinal do avaliador, o indivíduo levantava-se da cadeira o mais rápido possível. Foram três tentativas e ao final, calculada a média dos tempos (Rocha \& Guedes, 2013).

No teste de EE com controle visual, o indivíduo ficou em pé, com as mãos na cintura e olhando para frente. Ao sinal do avaliador, flexionava uma perna em 90 graus e tentava manter-se na posição por 30 segundos. Quando tocava o chão com o pé suspenso ou alcançava o tempo estabelecido, o teste era finalizado. Foram três tentativas com cada perna e o maior tempo foi utilizado (Spirduso, 2005).

Já na realização do teste $\mathrm{SR}$, o indivíduo sentava na ponta da cadeira, estendia uma perna enquanto a outra mantinha-se flexionada, buscava alcançar os dedos do pé da perna estendida, com uma mão sobreposta à outra. Permanecia nessa posição por dois minutos. Ao final, com uma fita métrica (marca Cescorf) mensurava-se a distância entre os dedos da mão e do pé. Quando ultrapassava a ponta do pé, foram considerados escores positivos, quando não, escores negativos. Quando as mãos chegavam na ponta do pé, era considerado escore zero (Jones et al., 1998).

No que diz respeito ao PEF utilizado, foram realizadas atividades de Condicionamento Aeróbio (CA) na forma de circuito, com obstáculos onde foram utilizados cones, arcos, steps, bastões e medicine ball. Para o monitoramento da intensidade foi utilizada a escala subjetiva de esforço de Borg (Borg, 1998). A tabela 1 mostra a rotina de CA realizado.

O Condicionamento Anaeróbio (CAN) foi realizado na sala de musculação. Foi utilizado o método global alternado por seguimento (Prestes, Foschini, Marchetti, Charro, \& Tibana,
2016). a tabela 2 apresenta detalhes do CAN realizado.

Tabela 1

Atividade de condicionamento aeróbio

\begin{tabular}{lc}
\multicolumn{2}{c}{ TREINAMENTO AERÓBIO INTERVALADO } \\
Estímulo & Tempo \\
\hline Caminhada (10-11 na escala de Borg) & $10^{\prime}$ \\
\hline Trote (12-13 na escala de Borg) & $4^{\prime} 30^{\prime \prime}$ \\
\hline Caminhada & $1^{\prime}$ \\
\hline Trote (12-13 na escala de Borg) & $4^{\prime} 30^{\prime \prime}$ \\
\hline Caminhada & $1^{\prime}$ \\
\hline Trote (12-13 na escala de Borg) & $4^{\prime} 30^{\prime \prime}$ \\
\hline Caminhada & 1 minuto \\
\hline Alongamento global & $30^{\prime \prime}$ cada posição \\
\hline
\end{tabular}

Tabela 2

Exercícios de condicionamento anaeróbio

\begin{tabular}{lcc}
\hline \multicolumn{1}{c}{ Exercício } & Séries & Repetições \\
\hline $\begin{array}{l}\text { Aquecimento na esteira com } \\
\text { velocidade progressiva (60 - 75\% }\end{array}$ & - & - \\
$\begin{array}{l}\text { FCM). Duração: } 10 \text { minutos } \\
\text { Agachamento com apoio na cadeira }\end{array}$ & 2 & 15 \\
\hline $\begin{array}{l}\text { Apoio de frente com apoio na } \\
\text { parede }\end{array}$ & 2 & 15 \\
\hline Extensão de tronco & 2 & 15 \\
\hline Remada (alta ou polia) & 2 & 15 \\
\hline Flexão plantar com apoio no step & 2 & 15 \\
\hline Flexão de cotovelos & 2 & 15 \\
\hline Abdominais no banco inclinado & 2 & 15 \\
\hline $\begin{array}{l}\text { Alongamento global. 30 “cada } \\
\text { posição }\end{array}$ & - & - \\
\hline
\end{tabular}

Ao longo do período de treinamento houve alterações nos exercícios mencionados no quadro 2, porém, foi priorizado os mesmos grupos musculares.

\section{Análise estatística}

O tratamento estatístico foi realizado com o software $\mathrm{R}$, versão 3.5.1. Para verificar a normalidade dos dados foi utilizada a estatística descritiva. Com isso, optou-se em utilizar o teste não paramétrico $p$ de Spearman para verificar a correlação entre as variáveis estudadas. Nesse teste, a correlação é considerada positiva quando as duas variáveis analisadas sobem de valor. É considerada negativa quando uma aumenta e a outra diminui. A correlação entre as variáveis pode ser considerada ausente, fraca, moderada ou forte. O nível de significância adotado foi $p \leq 0.05$.

\section{RESULTADOS}

No teste de VR, na primeira avaliação o idoso levou 1.29" para adotar a posição de pé. Nas demais avaliações, o tempo do idoso foi 0.65 ", 
1.54", 1.19", 1.17" e 1.48", respectivamente. A figura 1 mostra o seu desempenho.

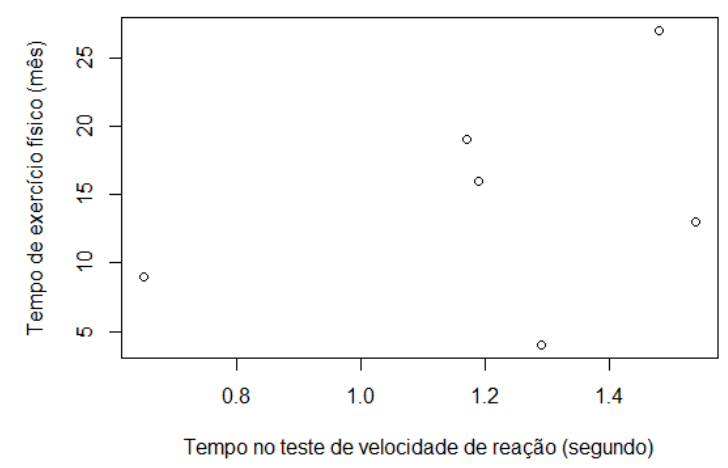

Figura 1. Desempenho do idoso no teste de velocidade de reação

Verificou-se uma não correlação negativa entre as variáveis tempo de exercício físico e tempo para o idoso adotar a posição em pé (+ $0.14),(p=0.78)$.

No que se refere o teste de EE com controle visual (Spirduso, 2005), na primeira avaliação o idoso permaneceu por 8.73" na posição unipodal. Nas demais avaliações, permaneceu respectivamente 11.16", 13.3", 11.34", 12.67" e $10.8 "$, como mostra a figura 2.

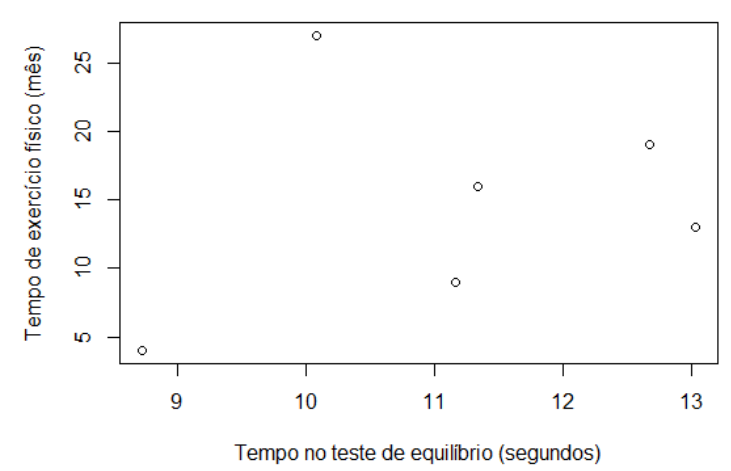

Figura 2. Desempenho do idoso no teste de equilíbrio estático

Houve uma fraca correlação positiva entre as variáveis tempo de exercício físico e equilíbrio (+ $0.25)$. Não foi estatisticamente significante ( $p=$ $0,62)$.

No que diz respeito à flexibilidade, no teste sitand-reach (Jones et al., 1998), nas avaliações realizadas com a perna direita o idoso atingiu: $13,-5,+6,+2,+2$ e -12 centímetros, respectivamente. Já com a perna esquerda o idoso alcançou $-5,-5,+2,+2,+2,-16$ centímetros.
As figuras 3 e 4 apresentam o desempenho do idoso.

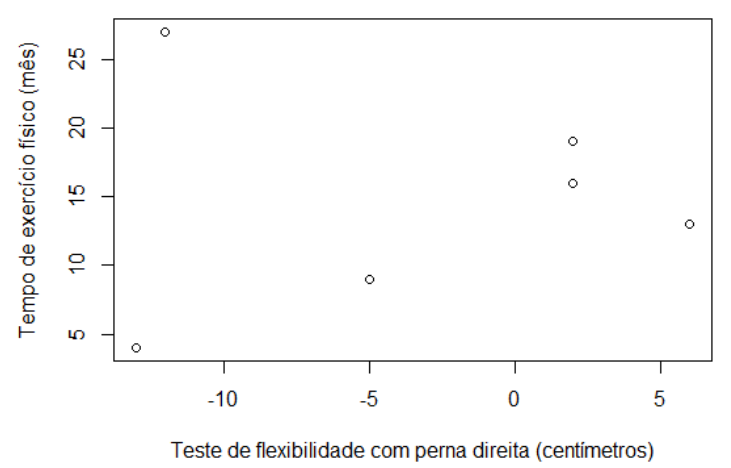

Figura 3. Desempenho no teste de flexibilidade com perna direita

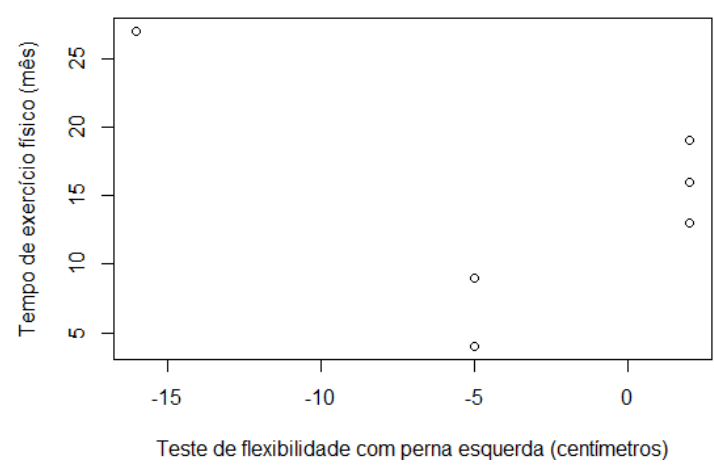

Figura 4. Desempenho no teste de flexibilidade com perna esquerda

Verificou-se uma correlação positiva fraca entre as variáveis tempo de exercício físico e flexibilidade perna direita $(+0.23)$, não foi estatisticamente significante $(p=0.65)$. Já com a perna esquerda houve uma ausência de correlação entre as duas variáveis estudadas (0.00), não foi estatisticamente significante $(p=1.00)$.

\section{DISCUSSÃO}

Com relação aos sintomas da DP, a bradicinesia segundo Guyton e Hall (2017) é um sintoma que dificulta a realização de movimentos iniciais por parte das pessoas acometidas por essa doença. De fato, o desempenho do idoso nos testes de velocidade de reação (Rocha \& Guedes, 2013) não foi satisfatório, efeito que também pode estar relacionado aos déficits de ativação muscular e sarcopenia, caracterizada pela redução progressiva e generalizada de massa muscular, especialmente as fibras tipo II, predispondo à insuficiência de força e potência do indivíduo (Fischer, 2014). 
No que se refere à flexibilidade osteomuscular, Segal, Hein e Basford (2004) a definem como a capacidade do músculo de mover um seguimento corporal por meio da sua amplitude de movimento. Uma característica dos indivíduos com DP é a rigidez muscular que, dificulta a realização dos movimentos. Contrapondo os resultados apresentados nesse estudo, os quais não evidenciaram uma evolução significativa do idoso nos testes de flexibilidade, em uma pesquisa desenvolvida por Reuter et all., (2011) com indivíduos com DP, os autores objetivaram avaliar os efeitos de um programa que envolvia flexibilidade e relaxamento no comprimento da passada. Concluíram que os exercícios utilizados melhoraram sua amplitude.

É bem verdade que em parte os resultados apresentados ficaram à quem do esperado, uma vez que não houve uma evolução considerável da capacidade funcional (equilíbrio, flexibilidade e velocidade de reação) do idoso. Contudo, considerando o fato da DP apresentar-se como uma condição crônica e degenerativa com seus efeitos adversos aumentando o declínio funcional, é provável que as atividades desenvolvidas favoreceram para retardar a progressão desfavorável dos sinais/sintomas dessa condição.

Por sua vez, McNeely, Mai, Duncan, e Earhart (2015) realizaram um estudo o qual teve como objetivo avaliar o equilíbrio de pessoas com DP participantes de um programa de atividade física envolvendo danças. Concluíram que a intervenção melhorou o equilíbrio dos indivíduos estudados.

Recentemente, Santos et all (2016) compararam o desempenho em equilíbrio e mobilidade funcional de pacientes com DP ativos e inativos. Participaram do estudo 41 indivíduos, 19 ativos e 22 inativos. O grupo ativo apresentou melhor desempenho na mobilidade funcional, porém não foi encontrada diferença significativa no equilíbrio entre os grupos estudados.

\section{CONCLUSÕES}

De acordo com os resultados apresentados, o PEF demonstrou baixo impacto no desenvolvimento da capacidade funcional do indivíduo após a intervenção. Com isso, não podemos inferir que o protocolo usado na pesquisa contribua de fato para melhorar a capacidade funcional na DP. Dessa forma, novos estudos devem utilizar amostras e uma frequência semanal maior, a fim de estabelecer tratamentos que possam verdadeiramente interromper o declínio funcional dessa população.

\section{Agradecimentos: \\ Agradecemos o apoio da Universidade Estadual Vale do Acaraú (UVA) e ao participante da pesquisa.}

\section{Conflito de Interesses:}

Nada a declarar.

\section{Financiamento:}

Nada a declarar.

\section{REFERÊNCIAS}

Borg, G. (1998). Borg's perceived exertion and pain scales. Champaign, IL: Human Kinetics.

Borrione, P., Tranchita, E., Sansone, P., \& Parisi, A. (2014). Effects of physical activity in Parkinson's disease: A new tool for rehabilitation. World Journal Of Methodology, 4(3), 133-143. doi: 10.5662/wjm.v4.i3.133

Burke, R. E., Dauer, W. T., \& Vonsattel, J. P. G. (2008). A critical evaluation of the Braak staging scheme for Parkinson's disease. Annals of Neurology, 64(5), 485-491.

Chehuen, J., \& Neto, A. (2012). Metodologia da pesquisa científica: da graduação à pós-graduação. Curitiba, PR, Brasil: CRV.

Conselho Nacional de Saúde (2013). Resolução n 466, de 12 de dezembro de 2012. Aprova normas regulamentadoras de pesquisas envolvendo seres humanos. Brasília: Diário Oficial da União.

Fischer, B. L. (2014). Efeitos do treinamento de potência na força muscular de indivíduos com doença de Parkinson. Dissertação de Mestrado, Universidade de Brasília, Brasília, Brasil.

Galhardo, M. M. A. M. C., Amaral, A. K. F. J., \& Vieira, A. C. C. (2009). Caracterização dos distúrbios cognitivos na doença de Parkinson. CEFAC, $11(2), 251-257$

Gobbi, L. T. B., Barbieri, F. A., \& Vitório, R. (Org). (2014). Doença de Parkinson e exercício físico. Curitiba, PR, Brasil: CRV.

Guyton, A. C., Hall, J. E. (2017). Tratado de Fisiologia Médica. (13a ed.). Rio de Janeiro, Brasil: Elsevier.

Hoehn, M. M., Yahr, M. D. (1967). Parkinsonism: onset, progression, and mortality. Neurology, $\underline{17}(5), 427-427$ 
Jones, C. J., Rikli, R. E., Max J., Noffal, G. (1998). The reliability and validity of a chair sit-and-reach test as a measure of hamstring flexibility in older adults. Research Quarterly for Exercise and Sport, 69(4), 338-343. doi: 10.1080/02701367.1998.10607708

Limongi, J. C. P. (2001). Conhecendo melhor a doença de Parkinson: uma abordagem multidisciplinar com orientações práticas para o dia-a-dia. SP, Brasil: Plexus.

Mcneely, M. E., Mai, M. M., Duncan, R. P., \& Earhart, G. M. (2015). Differential effects of tango versus dance for PD in Parkinson disease. Frontiers In Aging Neuroscience, 7, 1-8. doi: 10.3389/fnagi.2015.00239

Morris, M. E. (2000). Movement disorders in people with Parkinson's disease: A model for Physical Therapy intervention. PhysTher, 80(6), 578-597.

Petzinger, G. M., Holschneider, D. P., Fisher, B. E., McEwen, S., Kintz, N., Halliday, M., . . . Jakowec, M. W. (2015). The effects of exercise on dopamine neurotransmission in Parkinson's Disease: Targeting neuroplasticity to modulate basal ganglia circuitry. Brain Plasticity, 1(1), 2939. doi: 10.3233/BPL-150021

Prestes, J., Foschini, D., Marchetti, P., Charro, M., \& Tibana, R. (2016). Prescrição e periodização de treinamento de força em academias. (2a ed.). RJ, Brasil: Manole.

Reuter, I., Mehnert, S., Leone, P., Kaps, M., Oechsner, M., \& Engelhardt, M. (2011). Effects of a flexibility and relaxation programme, walking, and nordic walking on Parkinson's Disease. Journal Of Aging Research, 2011, 1-18.

Rocha, A. C., Guedes, D. P., Jr. (2013). Avaliação Física Para Treinamento Personalizado, Academias $e$ Esportes: Uma Abordagem Didática, Prática e Atual. SP, Brasil: Phorte.

Rose, M. H., Løkkegaard, A., Sonne-Holm, S., Jensen, B. R. (2013). Improved clinical status, quality of life, and walking capacity in Parkinson's Disease after body weight-supported high-intensity locomotor training. Archives of Physical Medicine and Rehabilitation, 94(4), 687-692. doi: 10.1016/j.apmr.2012.11.025

Santos, P. C. R., Morais, L. C., Simieli, L., Silva, E. L., Vitório, R., Ferreira1, M. D. T. O., ... Gobbi1, L. T. B. (2016). Comparação do equilíbrio e da mobilidade funcional entre pacientes com doença de Parkinson ativos e inativos. Revista Brasileira de Atividade Física \& Saúde, 21 (6), 534-541.

Segal, N. A., Hein, J., Basford, J. R. (2004). The effects of pilates training on flexibility and body composition: An observational study. Archives of Physical Medical Rehabiltation, 85(12), 1977-81.

Souto, M. O. (2009). Era outono em Barcelona: O meu encontro com Mr. Parkinson. Niterói, RJ, Brasil: Pathernon.

Spirduso, W. W., Francis, K. L., Macrae, P. G. (2005). Physical Dimensions of Aging (2nd ed.). Champaign, IL: Human Kinetics. 